\title{
Solution robotisée de mesure 3D en ligne
}

Christophe Lemoine - Responsable Commercial

Metrologic Group, 6 Chemin du Vieux Chêne, France - www.metrologic.fr

\begin{abstract}
Bringing Automation and 3D Inspection together is key to efficiency and access to inline Inspection. Our Metrolog X4 i-Robot Solution is a technical breakthrough that allows to easily deploy CMM style analysis with the flexibility of Robot programming and ease of use in one single software. This is the answer to a new market focus goal from all industries: aerospace, automotive, energy...
\end{abstract}

\section{Présentation du concept}

Metrolog X4 i-Robot est une solution logicielle de pilotage de la cellule de mesure basée sur Metrolog, le logiciel d'inspection 3Duniversel mondialement reconnu pour ses performances sur tous types de pièces et sa capacité à s'interfacer avec tous les moyens de mesure 3D du marché.

La flexibilité de Metrolog qui permet d'adresser les principaux moyens de mesure associée à sa capacité à piloter un robot a donnée naissance à cette nouvelle solution : le robot déplace simplement le moyen de mesure 3D dans le volume de mesure, gain significatif sur les temps de contrôle, les temps de flux de pièces, tout en conservant la précision du moyen " portable » et une excellente répétabilité des trajectoires.

Les grands principes de l'installation sont les suivants :

- Piloter directement l'ensemble robot et moyen de mesure avec un seul logiciel.

- Précision basée sur le moyen de mesure et non sur la précision du robot.

- Possibilité de piloter un large panel de robots industriels.

- Parfaite intégration de tous les types de moyens de mesure 3D du marché : tracker laser, scanner optique, scanner à contact, photogrammétrie...

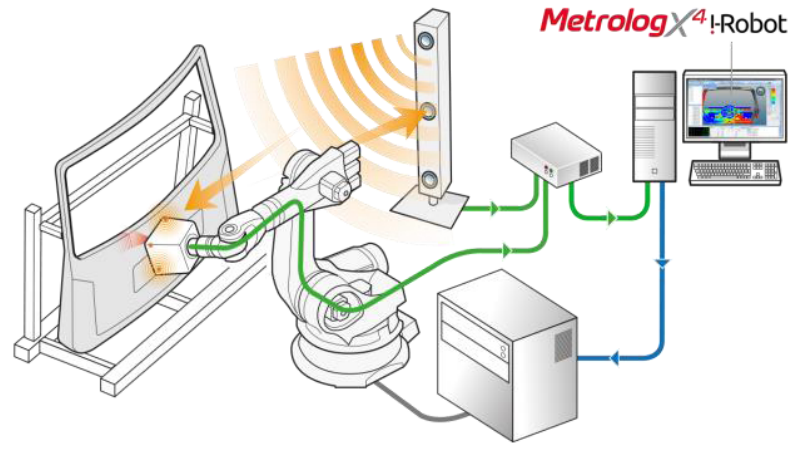

Figure 1. Solution de mesure robotisée par Metrolog X4 i-Robot

\section{Positionnement}

Cette solution est adressée à tous les industriels qui souhaitent automatiser leurs processus de mesure directement sur les lignes ou au plus proche de la ligne de production. Avec pour principal objectif d'optimiser les temps de mesure tout en conservant la précision de leurs systèmes actuels.

Les applications les plus fréquentes :

- Pièces de grand volume avec temps de contrôle conséquent (tous types de secteurs de fabrication) - Pièces de grandes séries (automobile par exemple) dans les flux de fabrication - Aéronautique (volume conséquent, contrôle à $100 \%)$.

\section{Intégration globale}

Metrolog X4 i-Robot permet d'associer pleinement le robot et le moyen de mesure $3 \mathrm{D}$ dans un seul logiciel.

La prise en charge est totale : gestion des axes rotatifs, linéaires supplémentaires, pilotage en parallèle d'un ou plusieurs robots, en liaison direct avec un système de supervision global si besoin.

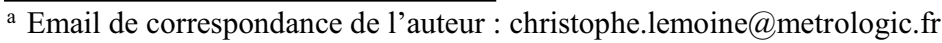


II s'agit d'une solution globale quelque soit la configuration et la complexité de la cellule de mesure.

\subsection{Intégration Robot}

Metrolog X4 i-Robot s'interface directement avec tous les contrôleurs robots (KUKA, Fanuc, ABB, Motoman, Stäubli...) et permet ainsi d'harmoniser les équipements robotiques au sein d'un site de production avec un seul logiciel de pilotage, d'acquisition des données de mesure et d'analyse dimensionnelle (incluant la partie rapport et export des résultats).

\subsection{Intégration de tous types de moyen de mesure 3D}

Metrolog X4 i-Robot offre le plus large choix de moyens de mesure avec plus de 85 interfaces développées à ce jour :

- Scanner optique

- Tracker laser

- Scanner à contact

- Caméra photogrammétrie

- Lumière blanche

- Laser radar

- Systèmes à ultra son et autres...

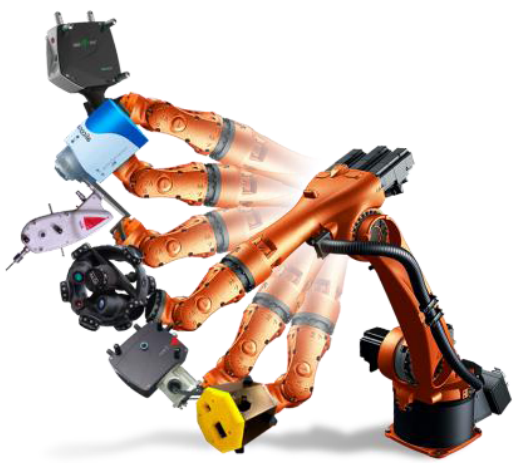

Figure 2. Flexibilité sur les moyens de mesure $3 D$ pris en charge

\subsection{Forte intégration dans le process client}

La ou les cellules de mesure équipées de Metrolog X4 i-Robot s'intègrent en ligne ou en proximité de cette dernière et peuvent se connecter au process global de supervision utilisé chez le client. Une même cellule peut également comporter plusieurs robots et gérer plusieurs types de technologies de mesure sur chaque robot, le logiciel inclus la gestion de changeurs d'outils pour démultiplier les possibilités d'intégration à l'infini. Les démarrages de cycle et les résultats de mesure effectués peuvent faire partie intégrante des process de mesure en place chez le client. Cette ouverture offre une réelle flexibilité au service de la fabrication assistée ou adaptée par la mesure.

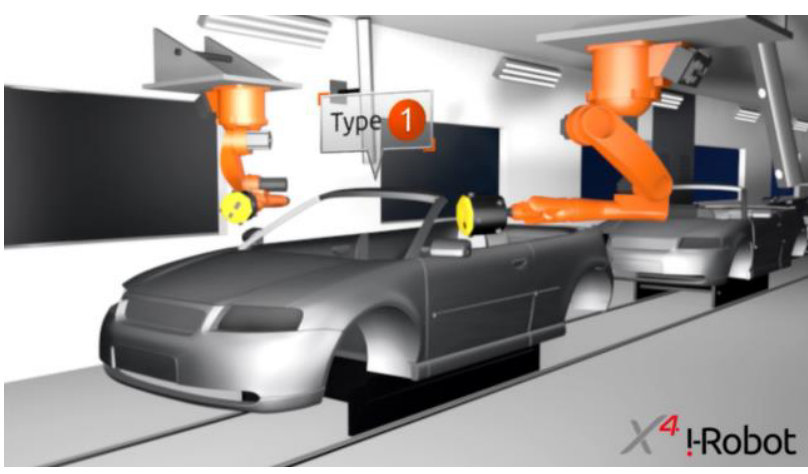

Figure 3. Solution de mesure en ligne

\section{Simulation}

L'intégralité de la cellule (robot, moyen de mesure, pièce et son support) peut être simulé en 3D au sein du logiciel pour la préparation des gammes de mesure en mode hors-ligne et en avance de phase. Ceci permet de préparer les programmes complets de déplacement du robot, l'acquisition dans un environnement virtuel sans prendre le risque d'endommager la cellule existante et d'effectuer les phases de mises au point sans avoir à interagir avec la ligne de production.

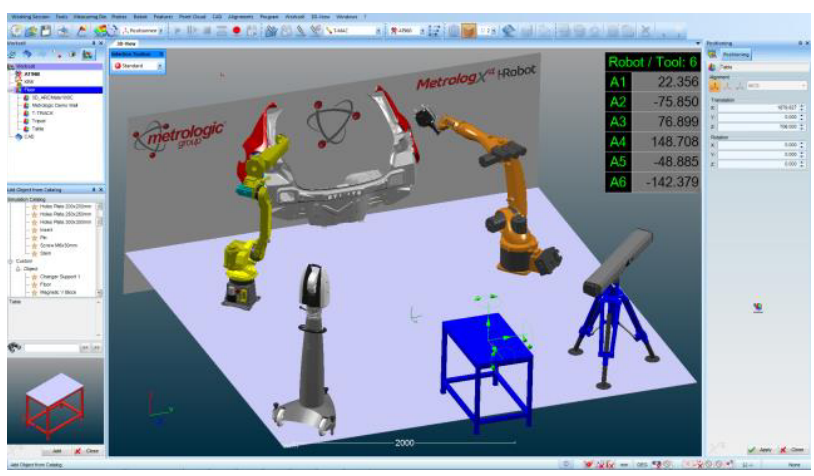

Figure 4. Solution logicielle de simulation de la cellule de mesure 